\title{
Research on Monitoring Information Management of Deep Foundation Pit Engineering Based on BIM Technology
}

\author{
Peng Wang ${ }^{1,2,3,4, *}$, Yan $\mathrm{Li}^{1,2,3,4}$ and $\mathrm{Na}$ Wang ${ }^{1,2,3,4}$ \\ ${ }^{1}$ Shaanxi Provincial Land Engineering Construction Group Co., Ltd., Xi'an, China. \\ ${ }^{2}$ Institute of Land Engineering and Technology, Shaanxi Provincial Land Engineering Construction Group Co., Ltd., Xi'an, China. \\ ${ }^{3}$ Key Laboratory of Degraded and Unused Land Consolidation Engineering, the Ministry of Natural Resources, China. \\ ${ }^{4}$ Shaanxi Provincial Land Consolidation Engineering Technology Research Center, China.
}

\begin{abstract}
With the rapid development of construction industry, there are more and more deep and large foundation pit projects with complex architectural environment and geological conditions. Deformation monitoring in deep foundation pit as a basic element of engineering quality assurance is becoming more and more important. At present, the management and expression form of monitoring data in deep foundation pit still stays in the stage of two-dimensional form and curve expression, and it can not reflect the deformation of deep foundation pit in real, fast and effective way.Take the 3\#, 4\# high-rise residential and underground garage project of a college in Xi'an as an example. Based on Revit software, taking BIM technology and Revit API as the theoretical basis, and taking Microsoft Visual Studio 2012 and Autodesk Revit 2014 as the development platform, we use $\mathrm{C} \#$ programming language to carry out the two development of information management module for deep foundation pit engineering monitoring. Combining BIM technology with monitoring data management of deep foundation pit, the information management mode of monitoring data of deep foundation pit is improved, which makes express expression of deep foundation pit construction monitoring more rapid and effective, and has guiding significance for safety construction of deep foundation pit engineering.
\end{abstract}

Key words: BIM; deep foundation pit engineering; monitoring management; Revit API; secondary development.

\section{Project overview}

With the rapid economic development, the construction scale of cities is getting bigger and bigger, and the number of super large-scale cities is also increasing. With the rapid growth of the urban population, in order to meet people's requirements for the living environment, the basements of high-rise and super high-rise buildings are getting deeper and bigger. According to the regulations, the excavation depth of more than 5 meters or the basement of three floors or more is called a deep foundation pit project, and its construction safety and construction quality must be strictly guaranteed.

Through the summary, analysis and research of many deep foundation pit projects, we can know that the combination of deep foundation pit engineering theory, construction experience and deformation monitoring during construction is the way to correctly guide the design and construction of deep foundation pit engineering. For large and medium-sized deep foundation pit projects with relatively complicated geological conditions or deep foundation pit projects with strict requirements on the environmental impact of surrounding buildings, it is often difficult to draw lessons from the same or similar deep foundation pit engineering construction experience, and it is also difficult to be theoretically accurate Quantitative analysis and prediction of the deformation of deep foundation pits. If the construction plan of a deep foundation pit project is designed and determined only based on the survey report before construction and the laboratory's geotechnical test parameters, there are too many uncertain factors. Through the summary and analysis of a large number of deep foundation pit engineering accidents, it can be seen that some units always believe that the foundation pit project as a temporary project does not need to invest too much capital and manpower, and ignores the importance of deformation monitoring during the construction of deep foundation pit projects. In the end, serious engineering accidents were caused. Common accidents such as the instability of foundation pit supporting structure, uneven settlement of surrounding buildings and roads, destruction of underground pipelines and facilities, and even casualties. Therefore, the deformation monitoring of the soil properties, the supporting structure of the foundation pit, and the surrounding building road environment during the construction process has obviously become an indispensable part of the construction process of the deep foundation pit engineering, and the deformation monitoring of the deep foundation pit engineering is regarded as An information technology is a necessary measure to ensure the quality of construction while avoiding accidents. Carrying out rigorous deformation monitoring for deep foundation pit projects is also accumulating valuable experience for the design and construction of more complex deep foundation pit projects.

It is proposed to build 3\#, 4\# high-rise residential buildings and underground garages of a college in Xi' an, which are located in the urban area of Xi'an, Shaanxi

\footnotetext{
*orresponding author: 694298560@qq.com
} 
Province, adjacent to the secondary main road of the city to the south. The terrain of the proposed site is relatively flat, the geomorphic unit is Huangtuliangwa, and the project \pm 0.00 corresponds to an absolute elevation of $419.90 \mathrm{~m}$. The plane size of the foundation pit: $67.275 \mathrm{~m}$ at the widest point from east to west and $57.06 \mathrm{~m}$ at the widest point from north to south. The perimeter of the bottom line of the foundation pit is $248.67 .0 \mathrm{~m}$, the actual side slope height of the foundation pit is about $7.0 \mathrm{~m}$, the garage is about $12.12 \mathrm{~m}$, and the local area is about $14.12 \mathrm{~m}$. The elevation of the excavation base of the foundation pit is $-7.37 \mathrm{~m}$ for the main building and $-13.27 \mathrm{~m}$ for the garage. It is planned to build $3 \#$ and $4 \#$ high-rise residential buildings with 33 floors and 1 basement.

\section{Engineering model creation}

Establish a BIM model based on the CAD drawings provided by the construction party, and import the CAD drawings into the Revit software to assist in modeling before creating the model. There are two ways for Revit software to import CAD drawings: The first way is to directly import the selected CAD drawings into Revit as the base drawing of the created model through the "Import CAD" command. This command is suitable for situations where you are not sure whether the CAD drawings are modified; The second is to import CAD drawings into Revit through the "Link CAD" command. This command is suitable for CAD design changes. If the source file is changed, the linked CAD drawing will also be changed accordingly. Once the source file is deleted or its location If there is a change, the corresponding CAD base drawing in Revit will be lost.

Step 1: CAD construction plan processing and import. This project uses the "Import CAD" command to import drawings as a base map for creating a deep foundation pit model. Since general CAD drawings are the basis for construction, component annotations, text descriptions, etc. are more detailed and complicated. In the Revit modeling process, these complex instructions will increase the burden on computer hardware, and the software will freeze or even crash, which will seriously affect Modeling efficiency. Therefore, before importing CAD drawings, delete unnecessary text, lines, and blocks, leaving only the axis and key nodes, and perform efficient modeling by automatically capturing the axis and key points. Use the "Import CAD" command in Revit to import the processed CAD construction plan. The import unit is uniformly selected in millimeters to facilitate project management.

Step 2: Positioning and creation of the deep foundation pit model. The ground surface is established through site options. The geological model is created using the floor slab in the Revit model. Five types of floor slabs with different thicknesses are created according to the engineering survey report, and different material attributes are assigned to replace the five different soil qualities within the excavation range of the foundation pit. Use the imported CAD drawings to locate the excavation boundary of the foundation pit, and use the building floor option to set the elevation to negative to create the excavated foundation pit model.

Use the "Pit Family Library Plug-in" to quickly load specific foundation pit engineering supporting structure families such as slope protection piles, plain soil compaction piles, and anchor rods. Here is how to display a pop-up window from which to select and apply the AIP Proceedings template paragraph styles.

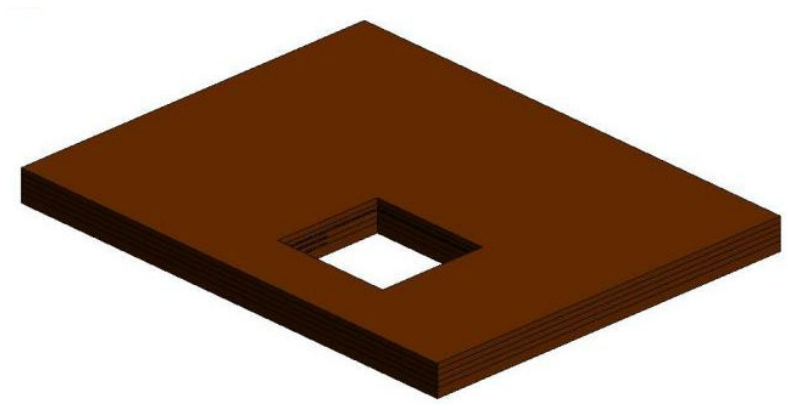

Figure 1. Soil model

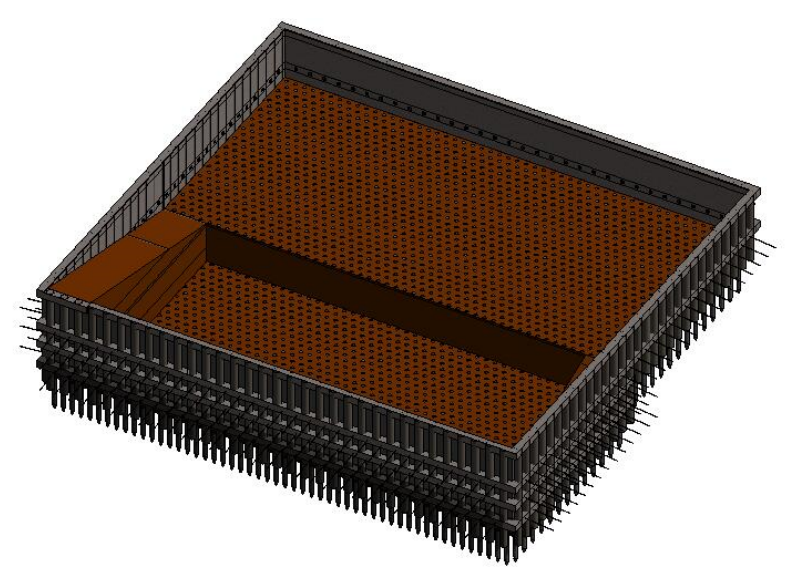

Figure 2. Foundation pit support structure

Step 3: Create a model of the surrounding environment of the foundation pit project. After the foundation pit model is created, according to the positioning of the surrounding buildings in the construction plan, follow the model creation steps to create the surrounding environment model of the deep foundation pit project.

Step 4: Arrangement of deformation monitoring points. According to the regulations, the horizontal displacement monitoring points on the top of the foundation pit slope are arranged. The horizontal displacement monitoring points on the top of the foundation pit slope are arranged on the crown beam of the foundation pit slope support structure, and are arranged along the periphery of the foundation pit. Monitoring points should be arranged at the sunny corners, the horizontal and vertical spacing of monitoring points should not be greater than $20 \mathrm{~m}$, and the number of monitoring points on each side should not be less than 3. According to the above requirements, a total of 16 foundation pit horizontal displacement monitoring points (JKJC1-JKJC16) are arranged in the foundation pit engineering model created above. The actual monitoring point layout should be appropriately adjusted according to the site conditions, and the layout should be made as far as possible when the requirements of the specification are 
met. The monitoring points are convenient for deformation observation.

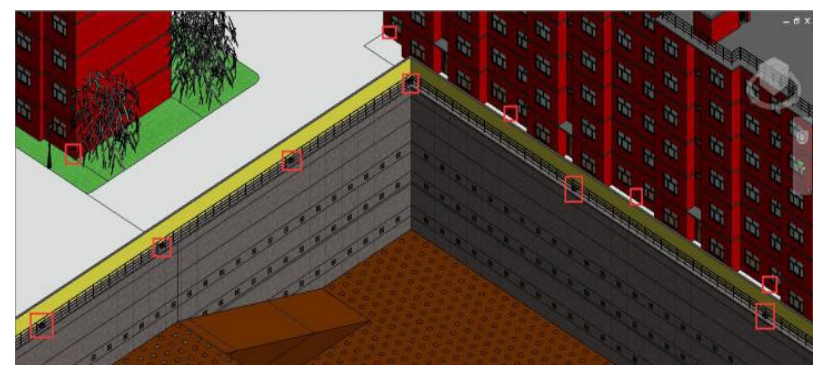

Figure 3. Monitoring point layout

Step 5: Parameterized setting of deformation monitoring points. After the layout of the deformation monitoring points of the foundation pit is completed, the location of the monitoring points shall be numbered according to the different monitoring items of the foundation pit engineering, and the early warning value and alarm value shall be set.

\section{Create a monitoring information management plug-in}

To realize the information management of deep foundation pit monitoring, the created plug-in program needs to realize the following functions: 1 . The plug-in can identify and extract the point number of the deformation monitoring point in the three-dimensional deep foundation pit model; The deformation monitoring data of the deep foundation pit is imported into the deep foundation pit model, and the monitoring date and monitoring data in the Excel file can be recognized; 3 . The plug-in contains an algorithm for processing the deformation monitoring data of the deep foundation pit, and the deformation of the deep foundation pit is calculated by the algorithm 4 . If the deformation of the monitoring point exceeds the specified value, the deformation of the monitoring point will be displayed in the form of a dialog box and an alarm interface will pop up; 5. Using the visual advantages of BIM technology, deep The color of the excessively deformed point in the foundation pit model changes in the real view mode. Perform programming design on the secondary development platform. The specific steps are as follows: Step 1: Create a new class library project with the help of Visual Studio 2012 environment, and rename the project as "Monitoring Management". After creating the project, you need to reference the interface group files Revit API.dll and Revit APIUI.dll in Revit. After the reference is successful, change the "copy local" in the reference properties of these two files to flase, otherwise a large number of .dll files will be copied Go to the run directory. The purpose of citing these two interface group files is to extract the interface for access to the Revit API.

Step 2: Add the reference to the namespace needed to write the plug-in program. The namespace is actually a specific memory area named by the developer. According to the specific needs of the user's secondary development, some named space domains are specified, and the global entities of the monitoring and management plug-in programming are placed in each namespace to prevent Confused with other global entities.

The namespace cited in this article mainly includes Revit API, Windows Forms controls, and input and output streams. The programming statements are as follows: using System; using System.Collections.Generic; using System.Linq; using System.Text; using System.Threading.Tasks; using Autodesk.Revit.DB; using Autodesk.Revit.UI; using Autodesk.Revit.UI.Selection; using Autodesk.Revit.ApplicationServices; using Autodesk.Revit.DB.Structure; using System.Windows.Forms;

Step 3: Add command attributes to the main program, set the update mode and transaction mode of the control command before writing the main program of each function. The programming statement is as follows:

[Autodesk.Revit.Attributes. Transaction(Autodesk.Revit. Attributes.TransactionMode.Manual)]

[Autodesk.Revit.Attributes.regeneration(Autodesk.Revit. Attributes.regenerationOption.Manual)]

The main part of this program is an external command, which is implemented by creating a new class derived from the IEternal Command interface and reloading the IExteral Command. execute() method.

Step 4: Write the main program that realizes each function. After the plug-in is running, it needs to be able to identify the point number of the deformation monitoring point in the deep foundation pit engineering model.

Step 5: After the completion of the main behavior program, the Visual Studio 2012 platform is required to compile the source code. If the source code fails to compile, return to the program to modify the problem code; if the compile succeeds, the software automatically generates the plug-in program and test results.

Step 6: After the compilation is successful, load the plugin into the Revit software. The program is successfully compiled, VS2012 will automatically generate test results and plug-in programs, start the Revit software to open the deep foundation pit model containing complete model information, use Revit's add-on module function to load the generated plug-in file into the software, and load and run on the model Plug-in program.

This is the paragraph spacing that occurs when you use the [ENTER] key.

\section{Application of monitoring management module}

The horizontal displacement monitoring data of the foundation pit of this project is measured by a total station. All the monitoring data in txt format will be exported through the computer, and the monitoring data will be extracted and exported to an Excel table. The application plug-in is loaded in the following order: "Add-on module" $\rightarrow$ "External tools" $\rightarrow$ "Add-In Manage (Manual) " $\rightarrow$ 
"Loaded commands " 、 "Loaded Application" $\rightarrow$ "Monitoring management.dll。

Click on "Run" Button The displacement monitoring dialog box pops up, select "Data", click the "Import xl" button to import the monitoring data into the plug-in; select "Control value" to input the deformation control value of the deep foundation pit project's horizontal displacement of $15 \mathrm{~mm}$, speed $2 \mathrm{~mm}$, The warning value is $10 \mathrm{~mm}$. After all the data and control values are loaded, click the "Apply" button. If the deformation monitoring data is normal and the deformation of each monitoring point does not exceed the deformation control value, no dialog box will pop up to prompt; if the data of the monitoring point exceeds the deformation Control value, a warning dialog box will pop up, and the cumulative displacement of the monitoring point and the deformation rate at the two monitoring time points will be displayed at the same time.

Close the warning dialog box and return to the 3D view mode of the foundation pit model. According to the setting of the module program, when the deformation of the monitoring point exceeds the warning value, the color of the monitoring point changes to yellow. At this time, the monitoring points in the deep foundation pit model that exceed the warning value change from normal color to yellow as a warning. According to regulations, the entrusting unit shall be notified immediately, and the entrusting unit shall report to relevant departments such as design and construction and assist in analyzing the cause, and encrypt it at the same time. Monitoring to prevent accidents.

\section{Conclusion}

In this paper, the entry point is to improve the management expression of traditional foundation pit monitoring information, based on the secondary development process of Revit software, and based on Revit API technology, combining traditional foundation pit engineering monitoring data management with BIM technology. The information management of foundation pit engineering monitoring based on Revit software has been realized, and the conclusions obtained are as follows: (1) Create a BIM model to facilitate the construction personnel to understand the complex site conditions. Based on the specifications, fully consider the convenience and feasibility of the deformation monitoring process, reasonably arrange the deformation monitoring points, select the optimal monitoring route, and reduce the human error of the monitoring process .

(2) Through the analysis and research of RevitAPI, VS2012 and RevitSDK are used as secondary development tools, and Revit2014 is used as the platform to realize the rapid loading of parameterized families in deep foundation pit engineering; create a "monitoring management" plug-in to realize deep foundation pit Import, calculation and result display of engineering monitoring data in BIM model.

(3) Apply the foundation pit monitoring information management module to the actual project, use BIM technology to change the monitoring data management method of the deep foundation pit project, change the twodimensional table and curve representation form of the monitoring report, and display the monitoring in a threedimensional manner in the BIM model As a result, the information management of foundation pit engineering monitoring was realized, and the project feasibility of the foundation pit monitoring information management module based on BIM technology was verified.

\section{References}

1. Wilkins R, Bastin G, Chrzanowski A, et al. A FULLY AUTOMATED SYSTEM FOR MONITORING PIT WALL DISPLACEMENTS[J]. Mining Engineering, 2003.

2. Hashash Y M A, Liu L Y, Ghaboussi J, et al. New Technologies for Tracking Urban Excavation Progress and Estimating Deformations[C]//Geoshanghai International Conference. 2006:336-341.

3. Matveenko V P, Fedorova V A, Shardakov I N. Theoretical justification of the possibility of constructing a fiber-optic earth surface deformation monitoring system[J]. Mechanics of Solids, 2013, 48(5):520-524.

4. Vaziri A, Moore L, Ali H. Monitoring systems for warning impending failures in slopes and open pit mines[J]. Natural Hazards, 2010, 55(2):501-512.

5. Eastman, Charles|And Others. An Outline of the Building Description System. Research Report No. 50.[J]. Architectural Drafting, 1974:23.

6. Monteiro A, Martins J P. A survey on modeling guidelines for quantity takeoff-oriented BIM-based design[J]. Automation in Construction, 2013, 35(11):238-253.

7. Nederveen G A V, Tolman F P. Modelling multiple views on buildings $\underset{\sim}{\hat{2}}[\mathrm{~J}]$. Automation in Construction, 1992, 1(3):215-224.

8. Malherek E, Laing G. Finer Points of Building Information Modeling[J]. Heating/piping/air Conditioning Engineering, 2011.

9. Smith D K, Tardiff M. Building Information Modeling: A Strategic Implementation Guide for Architects, Engineers, Constructors, and Real Estate Asset Managers[M]. Wiley, 2009. 